\title{
Acceleration of Wound Healing by Gelatin Film Dressings with Epidermal Growth Factor
}

\author{
Akane TANAKA ${ }^{1)}$, Toshiaki NAGATE ${ }^{1)}$ and Hiroshi MATSUDA ${ }^{1) *}$ \\ ${ }^{1)}$ Laboratory of Veterinary Molecular Pathology and Therapeutics, Division of Animal Life Science, Graduate School, Institute of \\ Symbiotic Science and Technology, Tokyo University of Agriculture and Technology, Fuchu, Tokyo 183-8509, Japan
}

(Received 18 March 2005/Accepted 20 May 2005)

\begin{abstract}
We examined in vivo efficiency of a gelatin film sheet with epidermal growth factor (EGF) for a novel therapeutic device for cutaneous wound repair. NIH3T3 fibroblasts and PAM212 keratinocytes proliferated when the cells were incubated with the homogenate of EGF containing gelatin sheets, indicating that the gelatin sheet retained biologic activity of EGF in the process of sheet formation. To evaluate therapeutic effects, the EGF containing gelatin sheets or control sheets were applied onto partial-thickness skin wounds made on dorsa of hairless dogs. Wound closure in wounds treated with EGF containing gelatin sheets was accelerated when compared to that of wounds treated with control sheets, exhibiting earlier reepithelialization of the epidermis and highly regulated repair of extracellular matrix in the dermis. Therefore, we concluded that the gelatin film is a useful material to keep EGF stable and the EGF containing sheet has the ability to become an efficient therapeutic agent for superficial or deep partial-thickness wounds in the skin. KEY WORDS: cytokine, gelatin sheet, reepithelialization, skin.
\end{abstract}

J. Vet. Med. Sci. 67(9): 909-913, 2005

Proper and immediate care of skin wounds is important for prevention of microbial infection and trans-epidermal water loss, leading to acceleration of wound repair. Recent findings have shown that moist condition accelerates wound healing when compared to dry condition [3, 13, 19, 21, 22]. The idea of keeping moisture during the process of wound healing has become general and has still been making progress today [21]. To keep optimal condition, the wound usually requires dressings or covering made of proper materials. These materials are expected to cover the whole wound area, to protect damaged tissue, and to stimulate the healing process. A multi-layer wound dressing is constructed with an elastic external layer, which serves as an artificial epidermis with an underlying layer made of hydrogels. Yannus, et al. [22] first developed a bilayer polymeric membrane that comprises of a silicone elastomer and a porous cross-linked network of collagen and glycosaminoglycan. Since then, various modifications have been made on development of bilayer wound dressings [13, 19]. Findings from many studies clearly indicate that healing of semi- or full-thickness wounds covered with dressings was accelerated when compared to those of uncovered controls $[3,13,19,22]$.

Epidermal growth factor (EGF) is a 53-amino-acid polypeptide that was first isolated from the mouse submaxillary gland [7, 17, 18]. EGF facilitates epidermal cell regeneration and plays an essential role in the process of wound healing through stimulation of proliferation and migration of keratinocytes [1, 16]. In addition, EGF not

\footnotetext{
* Correspondence to: Matsuda, H., Laboratory of Veterinary Molecular Pathology and Therapeutics, Division of Animal Science, Graduate School, Institute of Symbiotic Science and Technology, Tokyo University of Agriculture and Technology, 3-5-8 Saiwai-cho, Fuchu, Tokyo 183-8509, Japan.
}

only promotes formation of granulation tissue but also stimulates fibroblast motility that induces cell-cell adhesive properties consistent with an epithelial phenotype, thereby enhancing the early wound closure $[5,10]$. Topical application of ointment with EGF to full-thickness wounds made in rabbit's ears enabled the early repair of the wound [8]. Further, application of a silver sulfadiazine cream with EGF has brought significant acceleration of epidermal regeneration at the sites of grafted skins in 12 human patients [2]. The cytotoxicity of silver sulfadiazine impaired wound healing, while the addition of EGF could reverse the impairment and accelerate wound healing [6]; and this beneficial reaction of EGF requires a continuous exposure of the wound to EGF during the healing process evading the proteolysis of EGF by various proteases activated in the affected site $[4,6]$. However, instability of EGF in dressings because of its physical and chemical property brings a difficulty for a clinical trial.

Gelatin is able to avoid proteolysis of EGF by proteases in the wound site [15]. Moreover, biodegradability of gelatin in the lesion is suitable to provide the sustained release of EGF $[15,20]$. In the current study, we used gelatin as a hydrogel matrix in a dehydrate form and succeeded in producing a novel gelatin film with EGF for a dressing of the epidermal wound. We clearly demonstrated the biologic activity of EGF included in the gelatin film by a cell proliferation assay using NIH3T3 fibroblasts and PAM212 keratinocytes. The EGF containing sheet accelerated reepithelialization of partial-thickness wounds created in the dorsal skins of hairless dogs. Thus, we concluded that a gelatin sheet with EGF might be a useful dressing for treatment of the wound that suffered a loss of vast epidermis, including burns and surgical excision of skins. 


\section{MATERIALS AND METHODS}

Cells and animals: NIH3T3 fibroblasts (clone 5611) were obtained from Japan Health Science Foundation (Osaka, Japan; \#JCRB0615). PAM212 keratinocytes were provided from Dr. Katayama (Tokyo Medical and Dental University School of Medicine, Tokyo, Japan). Male hairless dogs were purchased from NOSAN Corporation (Tokyo, Japan). All experiments with animals were complied with the standards in the guidelines of the University Animal Care and Use Committee in Tokyo University of Agriculture and Technology.

EGF containing gelatin film: Five $\mathrm{g}$ of gelatin was added to $95 \mathrm{~g}$ of purified water and stirred at $40^{\circ} \mathrm{C}$ until dissolution. After cooled down to $30^{\circ} \mathrm{C}, 10 \mathrm{mg} / \mathrm{ml}$ human recombinant EGF (a kind gift from Higeta Shoyu Co., Ltd., Tokyo, Japan) was added and stirred until equilibrium. Gelatin with EGF was pour onto a polyester film and dried at $4^{\circ} \mathrm{C}$ for 3 days. The thickness of the film was adjusted to 10 $\mu \mathrm{m}$. The gelatin film included $20 \mu \mathrm{g}$ EGF in $10 \mathrm{~cm}^{2}$. The films were sterilized by gamma irradiation with $25 \mathrm{kGy}$. Stability of EGF sheets preserved under conditions at $4^{\circ} \mathrm{C}$, $25^{\circ} \mathrm{C}$, or $40^{\circ} \mathrm{C}$ for 1 month was measured by a quantikine human EGF ELISA kit (R\&D Systems, Minneapolis, MN, USA) according to the instructions.

Measurement of the EGF activity: The EGF containing sheet was cut into pieces $5 \mathrm{~mm} \times 10 \mathrm{~mm}$ large, dissolved in phenol red free $\alpha$-minimum essential medium ( $\alpha$-MEM, GIBCO Laboratories, Grand Island, NY, U.S.A.) containing $0.1 \%$ bovine serum albumin (Sigma Chemical Co., St. Louis, MO, U.S.A.) at $37^{\circ} \mathrm{C}$ for $10 \mathrm{~min}$. Because NIH3T3 fibroblasts react to EGF through EGF receptors [11], we used the cells for evaluation of biologic activity of EGF. NIH3T3 fibroblasts cultured in phenol red free $\alpha$-MEM supplemented with 10\% fetal bovine serum (FBS; Filtron, Brooklyn, Australia) and antibiotics in each well of 96-well plates were applied with various concentrations of the EGF sheet solution or recombinant human EGF. We also used PAM212 keratinocytes to elucidate activity of EGF films on reepithelialization. PAM212 keratinocytes were rendered quiescent by incubation in serum-free Dulbeco's modified Eagle's medium (DMEM; GIBCO Laboratories) containing $0.2 \%$ bovine serum albumin (Sigma Chemical) using 96well culture plates. Twelve hours later, culture medium was replaced to phenol red free DMEM containing 2\% FBS and antibiotics with or without various concentrations of the EGF sheet solution or recombinant human EGF. After incubated for $20 \mathrm{hr}, 10 \mu \mathrm{l}$ of $5 \mathrm{mg} / \mathrm{ml}$ 3-(4,5-Dimethyl-2-thizaolyl)-2,5-diphenyl-2H-tetrazolium Bromide (MTT) dissolved in PBS was added to each well and incubated for further $4 \mathrm{hr}$. The reaction was stopped by adding $100 \mu \mathrm{l}$ of $10 \% \mathrm{SDS}$ in $0.01 \mathrm{~N} \mathrm{HCl}$. The absorbance was measured at $577 \mathrm{~nm}$ with ImmunoMini NJ-2300 (Nalge Nunc International K.K., Tokyo, Japan).

In vivo evaluation of the EGF containing gelatin sheet: Hairless dogs were pre-medicated by subcutaneous injection of atropine sulfate $(0.04 \mathrm{mg} / \mathrm{kg})$ and xylazine hydro- chloride $(0.5 \mathrm{mg} / \mathrm{kg})$, subsequently anesthetized by intravenous injection of thiamylal sodium $(10 \mathrm{mg} / \mathrm{kg})$ and maintained with $2 \%$ isoflurane inhalation. Dorsal skin was sterilized by $70 \%$ ethanol and partial-thickness skin wounds $(3 \mathrm{~cm} \times 3 \mathrm{~cm})$ were made by a surgical Free-hand Dermatome (KEISEI Medical Industrial Co., Ltd., Tokyo, Japan). Partial-thickness wound is superficial or deeper skin loss that involves the epidermis, dermis or both, which is present as an abrasion or erosion. We made four partialthickness skin wounds in two dogs. After the gelatin film sheets with or without EGF were applied to wounds, each

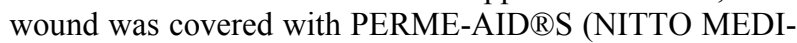
CAL Corp., Osaka, Japan) over the film sheet. The sheets were applied to each wound every day for 15 days and the conditions of the wound were recorded using a digital camera every third day. Areas of each wound were calculated by a digital image analysis using a Basic Quantifier (Genomic Solutions, Tokyo, Japan). The rate of wound healing was expressed in percentage using areas at day 0 as $100 \%$. Fifteen days later, skin biopsy specimens at the affected sites were obtained and fixed in $10 \%$ phosphatebuffered formalin. Paraffin sections ( $5 \mu \mathrm{m}$ thick) were stained with hematoxylin and eosin or with Masson trichrome staining for histological analysis. Each specimen was evaluated according to the criteria published by Matsuda, et al. [12]. Healing levels of the wound were graded as: - , none; \pm , slight; + , mild; ++ , moderate; +++ , considerable.

\section{RESULTS}

Quantification of immunoreactive EGF in the gelatin sheet: To evaluate the stability of EGF, immunoreactivity of EGF in the sheet was determined by an ELISA after preservation at $4{ }^{\circ} \mathrm{C}, 25^{\circ} \mathrm{C}$ and $40^{\circ} \mathrm{C}$ for a month (Table 1). More than $90 \%$ of immunoreactive EGF remained even after a long-term preservation at any condition we tested (Table 1).

Biologic activity of EGF in the gelatin sheet: To examine biologic activity of EGF in the gelatin sheet, we performed a bioassay of EGF using NIH3T3 fibroblasts. As shown in Fig. 1A, supernatants obtained from homogenates of the EGF containing sheet induced a dose-dependent prolifera-

Table 1. Effect of preservation for a month at each condition on immunoreactivity of EGF included in gelatin sheets

\begin{tabular}{cc}
\hline Preservation conditions & Remaining EGF (\%) \\
\hline Intact & 100 \\
$4^{\circ} \mathrm{C}, 1$ month & $93 \pm 6.2$ \\
$25^{\circ} \mathrm{C}, 1$ month & $96 \pm 4.9$ \\
$40^{\circ} \mathrm{C}, 1$ month & $99 \pm 5.8$ \\
\hline
\end{tabular}

Each EGF sheet was kept in a temperature constant box for a month at $4^{\circ} \mathrm{C}, 25^{\circ} \mathrm{C}$ and $40^{\circ} \mathrm{C}$, and immunoreactive EGF contents were measured by an ELISA. The data shows the percentage of EGF contents compared to that of the intact sheet. The data represent as the mean \pm SE of three different experiments. 
A

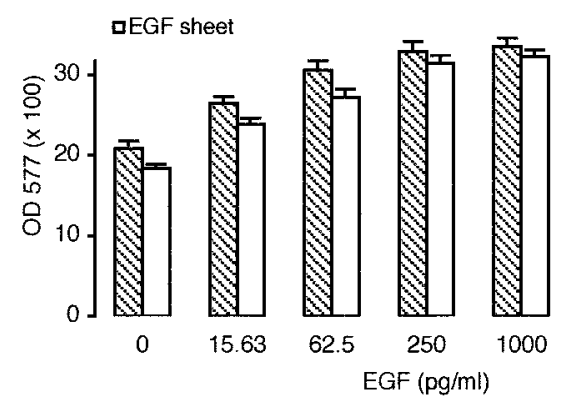

B

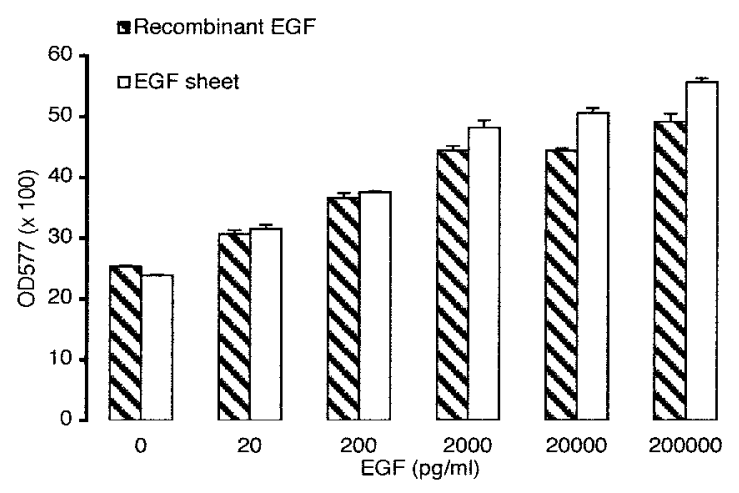

Fig. 1. Evaluation of biologic activity of EGF included in gelatin sheets by a MTT assay using NIH3T3 fibroblasts and PAM212 keratinicytes. NIH3T3 fibroblasts (A) or PAM212 keratinocytes (B) were incubated with indicated concentrations of human recombinant EGF or EGF sheet solution at $37^{\circ} \mathrm{C}$ for $20 \mathrm{hr}$. Data represents the mean $\pm \mathrm{SE}$ of 4 (NIH3T3 fibroblasts) or 3 (PAM212 keratinocytes) separate experiments.

tion of NIH3T3 fibroblasts, and the effect was comparable to that of recombinant human EGF at the same concentrations. This effect of EGF extracted from the gelatin sheet reached maximum at the concentration of $1000 \mathrm{pg} / \mathrm{ml}$, and the optical density (OD) value at $577 \mathrm{~nm}$ increased to 1.5 fold higher from vehicle control (Fig. 1A). We also evaluated EGF activity on PAM212 keratinocytes using BrdU uptake as a marker of DNA synthesis. As indicated in Fig. 1B, PAM212 keratinocytes proliferated in a dose-dependent manner in response to recombinant human EGF or EGF sheet solution.

Effect of the gelatin sheet with EGF on healing of partialthickness wound created on the dorsal skins of hairless dogs: To evaluate the ability of the sheet for acceleration of wound repair, we treated partial-thickness skin wounds created on the back of hairless dogs topically by covering them with EGF containing gelatin sheets. Wound areas reduced earlier in wounds treated with the gelatin sheet with EGF than those treated with control sheets (Fig. 2A and B). Wound areas treated with EGF sheets were significantly
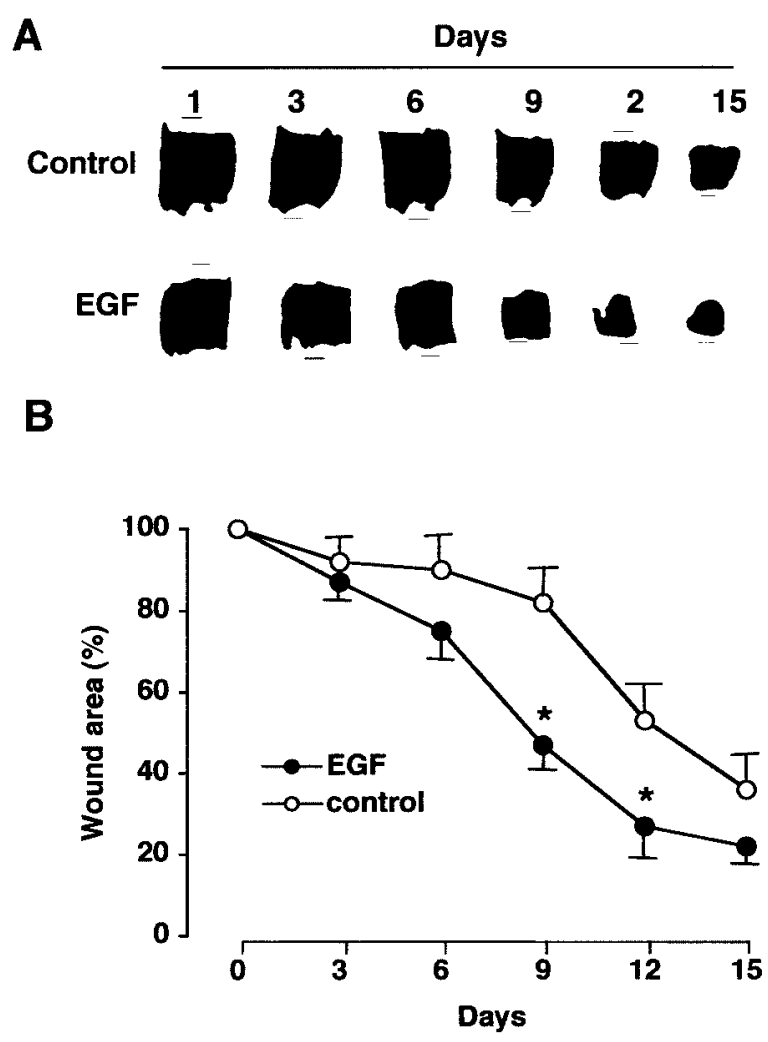

Fig. 2. Areas of each wound covered with gelatin sheets with or without EGF on dorsal skins of hairless dogs. Partial-thickness wounds were created on dorsal skins of hairless dogs by a Freehand Dermatome. Wounds were covered with EGF containing gelatin sheets or control sheets, and each sheet was replaced once a day for 14 days. The areas were measured by analyzing digital images using a Basic Quantifier (Genomic Solutions) (A) and the reduction in areas was displayed in B. The data represent as the mean \pm SE of 2 different hairless dogs with 2 wounds for each sheet.

decreased on day 9 and 12 than those treated with control sheets, and more than $50 \%$ of the area treated with EGF sheets was repaired on day 9 (Fig. 2B). Acceleration of wound healing was obvious in wounds treated with the EGF containing sheet especially in the early phase of the healing process (Fig. 2B).

Histological analysis revealed that the EGF containing sheet exhibited accelerating effects on parameters of wound healing, including complete reepithelialization and an increase in the degree of matrix density (Table 2 and Fig. 3). On the other hand, in wounds treated with control sheets, impairment in wound healing was evident in incomplete reepithelialization, low deposition of extracellular matrix, and continuous infiltration of neutrophils (Table 2 and Fig. 3 ). Wounds treated with control sheets were still consisted of homogeneous and unstructured tissues in the dermis (Fig. $3 \mathrm{~A})$; on the other hand, wounds treated with EGF containing sheets showed a precise layer structure of the dermis, reepi- 
Table 2. Histological evaluation of skin specimens obtained from affected sites of hairless dogs on day 15

\begin{tabular}{ccc}
\hline & Control sheet & EGF sheet \\
\hline Reepithelialization & \pm & +++ \\
Thickness of granulation tissue & ++ & + \\
Matrix density & \pm & ++ \\
Acute phase inflammatory cells & ++ & + \\
\hline
\end{tabular}

Biopsy specimens of wound tissue stained in hematoxylin and eosin were evaluated according to the criteria published by Matsuda et al. [12]. Wound healing levels were graded as: -, none; \pm , slight; + , mild; ++ , moderate; and +++ , considerable.

thelialization of epidermis, and basal membrane (Fig. 3B). Masson trichrome staining revealed an increase in collagen deposition in the dermis of wound treated with EGF containing sheets compared to low deposition of extracellular matrix of wound treated with control sheets (Fig. 3C and D).

\section{DISCUSSION}

Much is known regarding how EGF contributes to repair of skin wound by stimulating epidermal cell proliferation and migration [14]. On the other hand, dressings are effective on acceleration of wound healing by protecting wounds from infection and by keeping an environment of wounds with humidity $[3,13,19,20-22]$. Thus, the combination of EGF with dressings may provide a valid device for treatment of skin wounds [20]. Recent studies have attempted to produce dressings containing appropriate growth factors for wound healing, and their advantages have been discussed $[9,14,20]$. In this study, we used gelatin as a material of film dressings and succeeded to obtain an elastic, well-affixable and film-moldable material. Moreover, dried and sterilized gelatin sheets provided stable preservation of EGF for at least a month in various conditions. EGF included in the gelatin sheet retained a biologic activity, which could stim-

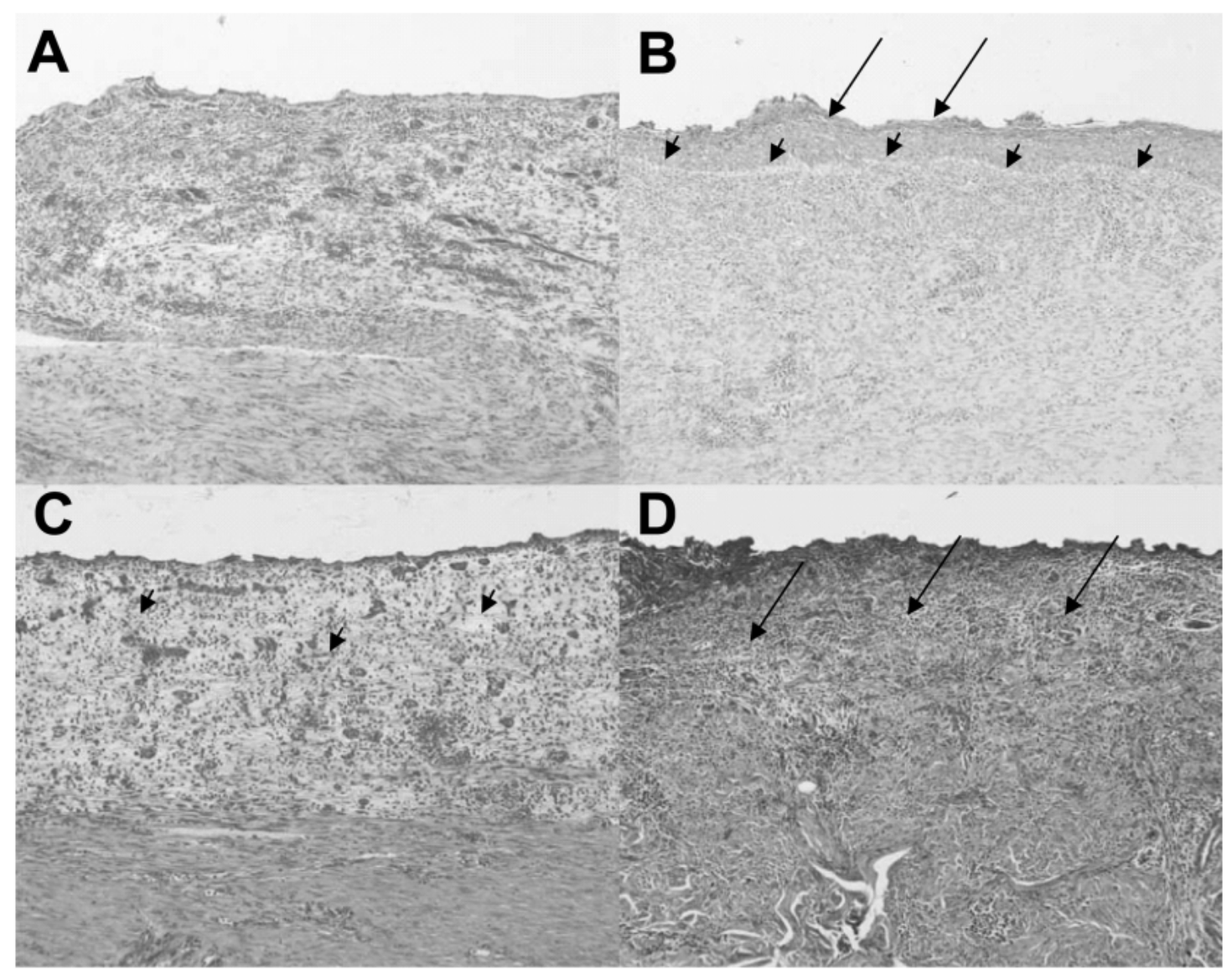

Fig. 3. Histological features of wound specimens from hairless dogs. After 15 days, biopsy specimens of wound tissues were removed, fixed in buffered formalin, and stained with Hematoxylin and eosin (A and B) or with Masson trichrome methods $(\mathrm{C}$ and $\mathrm{D})$. EGF containing gelatin sheets (B and D) or control sheets (A and C) were applied onto each wound once a day for 14 days. Wounds treated with EGF containing sheets showed a precise layer structure of the dermis, reepithelialization of epidermis (arrow), and basal membrane (arrow heads) (B). Masson trichrome staining revealed an increase in collagen deposition in the dermis of wound treated with EGF containing sheets (arrows) compared to low deposition of extracellular matrix of wound treated with control sheets (arrow heads) $(\mathrm{C}$ and D). Original magnification: $\times 80$. 
ulate proliferation of NIH3T3 fibroblasts and PAM212 keratinocytes. These results indicate that gelatin is a suitable material for production and preservation of dressings with active EGF. The high activity of EGF remained stable even after dried in gelatin films. Because a gelatin film that we developed is very thin and is easy to save, it may be useful for clinical use.

The local application of the gelatin sheet containing EGF onto partial-thickness of cutaneous wounds was effective to accelerate the rate of wound closure. Treatment with the EGF sheet induced early reepithelialization and highly regulated repair of extracellular matrix in dermis when compared to those of wounds treated with the control sheet, suggesting that EGF may not only promote keratinocytes proliferation and migration but also stimulate various functions of cells in epidermis to induce total repair of wounds in cooperation with other cytokines.

The present study clearly demonstrated that topical and continuous application of EGF included in gelatin sheets onto partial-thickness wounds accelerated wound repair, indicating that EGF containing gelatin sheets may have therapeutic potentialities for dressings of various skin wounds, including burns and surgical removal of skins.

ACKNOWLEDGEMENTS. We would like to thank NITTO DENKO Corp. (Osaka, Japan) for their technical support. We are also grateful to Higeta Shoyu Co., Ltd. (Tokyo, Japan) for providing human recombinant EGF. We greatly appreciate Dr. Atsuko Itakura for her technical support.

\section{REFERENCES}

1. Barrandon, Y. and Green, H. 1987. Cell migration is essential for sustained growth of keratinocyte colonies: the roles of transforming growth factor-alpha and epidermal growth factor. Cell 50: 1131-1137.

2. Brown, G.L., Nanney, L.B., Griffen, J., Cramer, A.B., Yancey, J.M., Curtsinger, L.J. 3rd., Holtzin, L., Schultz, G.S., Jurkiewicz, M.J. and Lynch, J.B. 1989. Enhancement of wound healing by topical treatment with epidermal growth factor. New Engl. J. Med. 321: 76-79.

3. Bruin, P., Jonkman, M.F., Meijer, H.J. and Pennings, A.J. 1990. A new porous polyetherurethane wound covering. $J$. Biomed. Mater. Res. 24: 217-226.

4. Buckley, A., Davidson, J.M., Kamerath, C.D., Wolt, T.B. and Woodward, S.C. 1985. Sustained release of epidermal growth factor accelerates wound repair. Proc. Natl. Acad. Sci. U.S.A. 82: 7340-7344.

5. Buckley, A., Davidson, J.M., Kamerath, C.D. and Woodward, S.C. 1987. Epidermal growth factor increases granulation tissue formation dose dependently. J. Surg. Res. 43: 322-328.

6. Cho-Lee, A.R., Leem, H., Lee, J. and Chan-Park, K. 2005. Reversal of silver sulfadiazine-impaired wound healing by epi- dermal growth factor. Biomaterials 26: 4670-4676.

7. Cohen, S. 1962. Isolation of a mouse submaxillary gland protein accelerating incisor eruption and eyelid opening in the new-born animal. J. Biol. Chem. 237: 1555-1562.

8. Franklin, J.D. and Lynch, J.B. 1979. Effects of topical applications of epidermal growth factor on wound healing. Experimental study on rabbit ears. Plast. Reconstr. Surg. 64: 766770.

9. Hong, S.R., Lee, S.J., Shim, J.W., Choi, Y.S., Lee, Y.M., Song, K.W., Park, M.H., Nam, Y.S. and Lee, S.I. 2001. Study on gelatin-containing artificial skin IV: A comparative study on the effect of antibiotic and EGF on cell proliferation during epidermal healing. Biomaterials 22: 2777-2783.

10. Kurten, R.C., Chowdhury, P., Sanders, R.C. Jr., Pittman, L.M., Sessions, L.W., Chambers, T.C., Lyle, C.S., Schnackenberg, B.J. and Jones, S.M. 2005. Coordinating epidermal growth factor-induced motility promotes efficient wound closure. Am. $J$. Physiol. Cell Physiol. 288: C109-121.

11. Kwan, R.W., Wong, R.W. and Chan, S.Y. 1999. Expression of full length or truncated epidermal growth factor precursor transforms NIH3T3 fibroblasts. Int. J. Oncol. 15: 281-284.

12. Matsuda, H., Koyama, H., Sato, H., Sawada, J., Itakura, A., Tanaka, A., Matsumoto, M., Konno, K., Ushio, H. and Matsuda, K. 1998. Role of nerve growth factor in cutaneous wound healing: accelerating effects in normal and healing-impaired diabetic mice. J. Exp. Med. 187: 297-306.

13. Matsuda, K., Suzuki, S., Isshiki, N., Yoshioka, K., Okada, T. and Ikada, Y. 1990. Influence of glycosaminoglycans on the collagen sponge component of a bilayer artificial skin. Biomaterials 11: 351-355.

14. Nanney, L.B. 1990. Epidermal and dermal effects of epidermal growth factor during wound repair. J. Invest. Dermatol. 94: 624-629.

15. Okumura, K., Kiyohara, Y., Komada, F., Iwakawa, S., Hirai, M. and Fuwa, T. 1990. Improvement in wound healing by epidermal growth factor (EGF) ointment. I. Effect of nafamostat, gabexate, or gelatin on stabilization and efficacy of EGF. Pharm. Res. 7: 1289-1293.

16. Rheinwald, J.G. and Green, H. 1977. Epidermal growth factor and the multiplication of cultured human epidermal keratinocytes. Nature (Lond.) 265: 421-424.

17. Savage, C.R. Jr., Hash, J.H. and Cohen, S. 1973. Epidermal Growth Factor. J. Biol. Chem. 248: 7669-7672

18. Savage, C.R. Jr., Inagami, T. and Cohen, S. 1972. The primary structure of epidermal growth factor. J. Biol. Chem. 247: 7612 7621

19. Suzuki, S., Matsuda, K., Isshiki, N., Tamada, Y. and Ikada, Y. 1990. Experimental study of a newly developed bilayer artificial skin. Biomaterials 11: 356-360.

20. Ulubayram, K., Nur-Cakar, A., Korkusuz, P., Ertan, C. and Hasirci, N. 2001. EGF containing gelatin-based wound dressings. Biomaterials 22: 1345-1356.

21. Winter, G.D. 1962. Formation of the scab and the rate of epithelization of superficial wounds in the skin of the young domestic pig. Nature (Lond.) 193: 293-294.

22. Yannus, I.V. and Burke, J.F. 1980. Design of an artificial skin. I. Basic design principles. J. Biomed. Mater. Res. 14: 65-81. 